\section{Retinal pigment epithelial changes and choroidal neovascularisation at the edge of posterior staphylomas; a case series and review of the literature}

Keywords: myopia; posterior staphyloma; retinal pigment epithelial atrophy; choroidal neovascular membrane

Introduction

Scleral ectasia from posterior expansion of eyeball results in posterior staphyloma, a condition that is usually associated with pathological myopia, but has also been described in eyes without significant myopia. The chorioretinal changes seen within these staphylomas have been well described, ${ }^{1,2}$ but to date there are few descriptions of the changes that occur at the edge of a staphyloma. We report five patients who showed changes at the edge of the posterior staphyloma in the macular region, four of who developed visual disturbance secondary to it.

\section{Case reports}

Case 1

A 16-year-old Caucasian male with severe astigmatism was referred querying macular dystrophy, as he presented with reduced visual acuity in his right eye not improving with glasses. He was known to be amblyopic in his left eye. On presentation, his best-corrected visual acuity was $6 / 12$ in both eyes. His refraction was $+3.5 \mathrm{DS} /-3.5 \mathrm{DC}$ at $45^{\circ}$ in the right eye and $+4.00 \mathrm{DS} /-3.5 \mathrm{DC}$ at $160^{\circ}$ in the left eye. Orthoptic assessment and anterior segment examination were normal. Fundus examination showed bilateral tilted discs with 

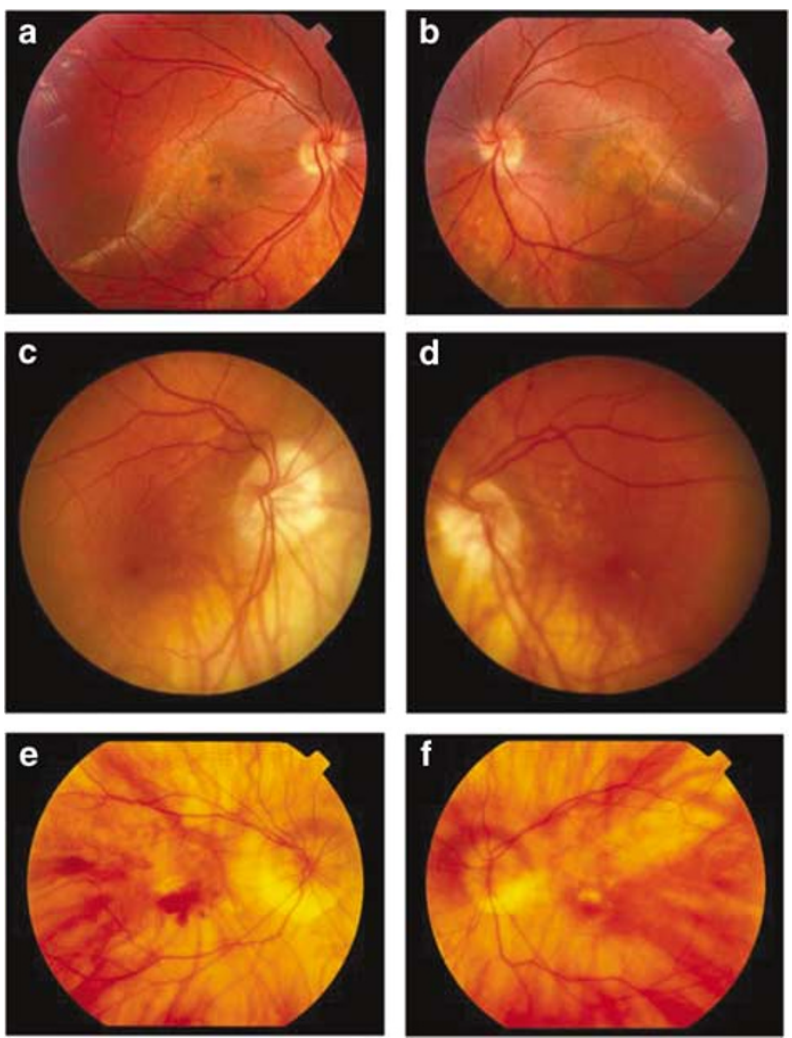

Figure 1 (a, b) Case 1-Right and left fundus showing RPE atrophy at the edge of the staphyloma. (c, d) Case 2-Right and left fundus showing tilted discs with heterotropia of the foveae and retinal pigment epithelial changes, at the edge of the staphyloma. (e, f) Case 4-Right and left fundus showing RPE atrophy, and a disciform lesion in the right eye, seen at the edge of the staphyloma.

inferior posterior staphylomas. Retinal pigment epithelial (RPE) atrophy was seen at both maculae at the edge of the staphyloma (Figure 1a and b). No changes were seen within the staphyloma.

Ultrasound examination confirmed the presence of posterior staphylomas in both eyes. Electro-diagnostic tests were normal. Fundus fluorescein angiogram showed transmission defects placed obliquely at both maculae within which were areas of spotty hyperfluorescence (Figure $2 \mathrm{a}$ and $\mathrm{b}$ ). The defects coincided with the point of change of curvature of the posterior staphyloma in both eyes. Some areas of brighter hyperfluorescence, probably representing confluent RPE atrophy were seen in the left eye. The patient is under regular review and has noticed no worsening of symptoms.

\section{Case 2}

An 80-year-old myopic lady who had previously undergone bilateral cataract surgery with good outcome
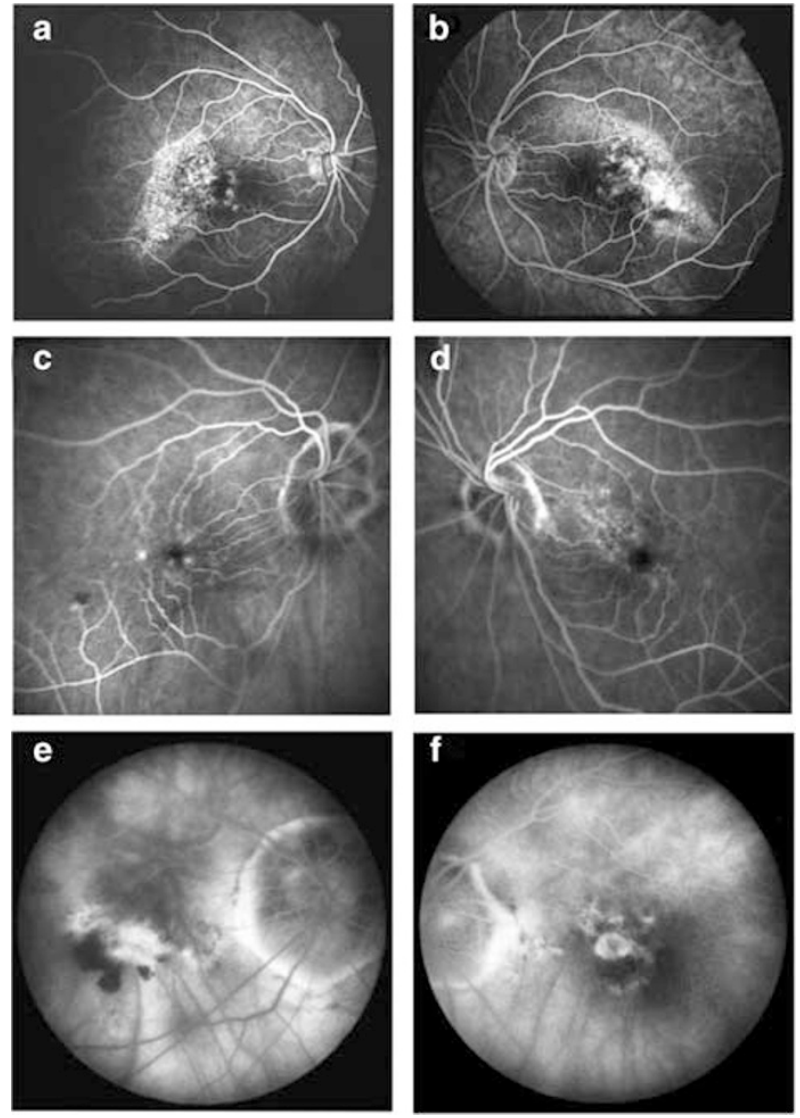

Figure $2(a, b)$ Case 1-Fundus flourescein angiogram of the right and left eye showing transmission defects seen obliquely at both maculae at the edge of the staphyloma with areas of spotty hyperfluorescence. (c, d) Case 2-Fundus flourescein angiogram of the right and left eye showing transmission defects suggestive of RPE atrophy at the edge of the staphyloma with areas of spotty hyperfluorescence around the fovea in the right eye. $(e, f)$ Case 4 -Fundus fluorescein angiogram of the right eye showing a choroidal neovascular membrane and the left eye showing areas of hyperfluorescence along the edge of the staphyloma.

was referred with deterioration of vision in the right eye over a few months. Her visual acuity at the time of presentation was $6 / 9$ in both eyes. Her original refractive error was $-6.50 \mathrm{DS} /+2.25 \mathrm{DC}$ at $164^{\circ}$ in the right eye and $-7.50 \mathrm{DS} /+1.25 \mathrm{DC}$ at $107^{\circ}$ in the left. Fundus examination showed bilateral tilted discs with heterotropia of the foveae. Both foveae appeared much lower than the optic discs (Figure 1c and d). Posterior staphyloma were noted in both eyes and were confirmed by ultrasound examination. RPE changes were seen, particularly in the left eye, at the edge of the staphyloma. No such changes were seen within the staphyloma. Fundus fluorescein angiogram showed spotty hyperfluorescence around the fovea in the right eye and along the edge of the staphyloma in the left (Figure 2c and $\mathrm{d}$ ). No choroidal neovascular membrane was demonstrable. 


\section{Case 3}

A 77-year-old male with high myopia was referred with complaints of monocular diplopia in his left eye following left cataract surgery. His original refractive error was $-7.75 \mathrm{DS} /-0.25 \mathrm{DC}$ at $79^{\circ}$ in the right eye and $-2.25 \mathrm{DS} /-1.50 \mathrm{DC}$ at $23^{\circ}$ in the left. He was known to have amblyopia in the right eye. On presentation, his best-corrected visual acuity was hand movements in the right and 6/9 in the left eye. The anterior segment examination was unremarkable with the intraocular lens implant in the bag and not displaced. Fundus examination revealed bilateral tilted optic discs with posterior staphyloma. Pigment irregularity was noted along the oblique edge of the staphyloma inferotemporal to the left optic disc. These changes were not seen within the staphyloma. Fundus fluorescein angiogram showed the edge of the staphyloma with pigment epithelial changes at the point of change of curvature in the left eye with no evidence of leakage or choroidal neovascular membrane.

\section{Case 4}

An 81-year-old myopic female presented with worsening of visual acuity in the right eye. She was known to be amblyopic in the left eye. On examination, her bestcorrected visual acuity was 6/18 in right eye and 6/60 in the left. Her refractive error was $-5.50 \mathrm{DS} /+0.25 \mathrm{DC}$ at $131^{\circ}$ in the right eye and $-5.50 \mathrm{DS} /+0.25 \mathrm{DC}$ at $134^{\circ}$ in the left. Anterior segment examination showed bilateral early cortical lens opacities. Fundus examination revealed a tilted disc in the right eye and bilateral staphylomas running horizontally from and extending over the top of both optic discs. RPE atrophy and a disciform lesion with surrounding haemorrhage were seen at the right macula at the edge of the staphyloma (Figure 1e and f). Myopic changes with RPE atrophy were seen within the staphyloma in both eyes. Fundus fluorescein angiogram (Figure 2e and $\mathrm{f}$ ) and ICG angiogram revealed features of an occult choroidal neovascular membrane in the right eye. No further treatment was planned.

\section{Case 5}

A 66-year-old myopic female presented with worsening of visual acuity in the right eye. She had bilateral cataract surgeries carried out 3 years ago prior to which, her refraction was $-15.75 \mathrm{DS} /+1.75 \mathrm{DC}$ at $11^{\circ}$ in the right eye and $-16.50 \mathrm{DS} /+0.50 \mathrm{DC}$ at $152^{\circ}$ in the left. On examination, her best-corrected visual acuity was $6 / 18$ in the right and 6/12 in the left eye. Fundus examination showed posterior staphyloma in both eyes, the edge of which was temporal to the fovea. RPE atrophy with adjacent subretinal haemorrhage was seen at the edge of the staphyloma in the right eye. Similar RPE changes were also seen in the left eye along with RPE atrophy within the staphyloma. Fundus fluorescein angiogram showed marked hyperfluorescence along the edge of the staphyloma secondary to the RPE atrophy in both eyes and a small classic disciform lesion at the right macula for which she has undergone photodynamic therapy. However, the visual improvement may be compromised due to the extensive RPE atrophy, which abuts the fovea. On review, 3 months later, the choroidal neovascular membrane was not leaking and she has maintained her vision at $6 / 18$. The patient is under regular review.

\section{Discussion}

High myopia is associated with progressive and excessive elongation of the globe and maybe associated with posterior staphyloma or ectasia of the posterior eye wall. The ectasia maybe associated with an edge of altered contour that passes close to or through the macula, either obliquely or horizontally. Posterior staphyloma has also been described in eyes without significant elongation of the globe. Various fundoscopic changes develop within posterior staphyloma. These include diffuse chorioretinal atrophy, patchy atrophy, RPE defects, lacquer cracks, and macular choroidal neovascularisation (CNV). In patients with high myopia, patchy atrophy, and lacquer cracks are especially important predisposing lesions in the development of CNV. ${ }^{1}$

Steidl and Pruett, ${ }^{2}$ in their review of the macular complications associated with posterior staphyloma, graded staphyloma formation from zero to four based on the contour changes determined from stereoscopic indirect ophthalmoscopy, stereo viewing of photographs and angiograms, and B scan ultrasonographic data. They found that there was an unexpected high incidence of choroidal neovascular membrane, haemorrhage, and poor best-corrected visual acuity in lower staphyloma categories. As choroidal neovascular membranes were more frequently seen in those eyes with less severe posterior staphylomas, the authors suggested that a wellpreserved choriocapillaris was a risk factor for the development of choroidal neovascular membranes. ${ }^{2}$

However, the authors did not specify where the chorioretinal changes that they described occurred in the staphyloma and, in particular, whether any of these changes occurred at its edge.

There are very few reports describing the chorioretinal changes that develop at the edge of posterior staphylomas. Tsuboi et $a l^{3}$ reported seven cases where a localised choroidal neovascular membrane developed at 
the upper edge of inferior posterior staphyloma. In each case the staphylomas were shallow and small in size, with the sharp upper edge lying across the macular area. Three of the cases with relatively low refractive power showed a focus of chorioretinal atrophy lying across the upper edge of the staphyloma extending into the macular area. The authors hypothesised that in each case, the sharp change in contour seen at the upper edge of the staphyloma may cause localised chorioretinal degeneration with secondary disruption of Bruch's membrane and subsequent $\mathrm{CNV}$ when the macular area is involved. ${ }^{3}$

Quaranta et $a l^{4}$ reported one case where multiple choroidal neovascular membranes developed along the inner edge of a macular staphyloma with pigmentary disturbance and focal chorioretinal atrophic patches in the macular region in a patient with pathological myopia. Based on this observation, the authors suggested that the edge of a staphyloma where the choriocapillaris is well preserved was an important transitional zone associated with an increased risk of chorioretinal changes and choroidal neovascular membrane formation.

Interestingly, the one patient in our series (Case 4) in whom an Indocyanine green angiogram was performed did show preservation of the choriocapillaris along the border of the staphyloma.

Chorioretinal changes, including choroidal neovascular membrane formation, have also been reported at the border of posterior staphylomas in patients with congenital tilted disc syndrome. ${ }^{5,6}$

We present five further cases where there is evidence of chorioretinal changes located on or around the edge of the staphyloma. These changes appear to be different from those within the staphyloma and they seem to coincide with the point of change of curvature. Two patients (cases four and five) developed a choroidal neovascular membrane at the edge. In one other case (case one), the chorioretinal changes were so marked that the patient was thought to have a macular dystrophy. Three of these patients (cases 1-3) were also noted to have bilateral tilted discs and one patient (case 4) was noted to have a tilted disc in the right eye. Thus, these changes may be seen in patients with myopia with tilted discs as well as in those with high myopia in general.

This case series illustrates the variety of changes that can occur at the edge of posterior staphylomas, ranging from RPE disturbance to choroidal neovascular membrane formation. The RPE changes seen at the edge of staphylomas can be marked and may be misinterpreted as a macular dystrophy. Our observations compare favourably with the findings of other similar case series and they support the hypothesis that the edge of a staphyloma is a transitional zone that has a high risk of secondary chorioretinal changes including choroidal neovasacular membrane formation.

\section{References}

1 Ohno-Matsui K, Yoshida T, Futagmi S, Yasuzumi K, Shimada N, Kojima A et al. Patchy atrophy and lacquer cracks predispose to the development of choroidal neovascularisation in pathological myopia. $\mathrm{Br} J$ Ophthalmol 2003; 87: 570-573.

2 Steidl SM, Pruett RC. Macular complications associated with posterior staphyloma. Am J Ophthalmol 1997; 123: 181-187.

3 Tsuboi S, Uchihori Y, Manabe R. Subretinal neovascularisation in eyes with localised inferior posterior staphylomas. Br J Ophthalmol 1984; 68: 869-872.

4 Quaranta M, Brindeau C, Coscas G, Soubrane G. Multiple choroidal neovascularizations at the border of a myopic posterior macular staphyloma. Graefe's Arch Clin Exp Ophthalmol 2000; 238: 101-103.

5 Prost M, DeLaey JJ. Choroidal neovascularisation in the tilted disc syndrome. Int Ophthalmol 1988; 12: 131-135.

6 Stur M. Congenital tilted disc syndrome associated with parafoveal subretinal neovascularisation. Am J Ophthalmol 1988; 105: 98-99. 